INPLASY

PROTOCOL

To cite: Du et al. Aerobic

Exercise Improve Cognition in

People with Schizophrenia: A

Meta-Analysis. Inplasy

protocol 2020100075. doi:

10.37766/inplasy2020.10.0075

Received: 21 October 2020

Published: 21 October 2020

Corresponding author:

Liu Wei

1435651603@qq.com

Author Affiliation:

The First Affiliated Hospital of Harbin Medical University

Support: None.

Review Stage at time of this submission: Preliminary searches.

Conflicts of interest: None.

\section{Aerobic Exercise Improve Cognition in People with Schizophrenia: A Meta-Analysis}

\author{
Du, J1; Zheng, Y2; Liu, W3.
}

Review question / Objective: This meta-analysis of randomized controlled trials aim to evaluate the efficiency of adjunctive aerobic exercise for cognition in people with schizophrenia.

Condition being studied: Schizophrenia is one of the severe mental diseases. The prevalence rate of schizophrenia in the world is $0.5 \% \sim 1 \%$. In China, its lifetime prevalence rate is about $0.7 \%$, bringing a substantial economic burden to families and society. Cognitive dysfunction is one of the core symptoms of schizophrenia. It often appears before the psychotic symptoms such as hallucinations and delusions and runs through the disease's whole course. However, there is still a lack of effective drugs to improve cognitive function. In recent years, some studies have shown that aerobic exercise (AE) can improve the cognitive function of schizophrenic patients. After long-term aerobic exercise, the volume of the temporal lobe and hippocampus increases, and the content of brain-derived neurotrophic factor in serum increased. Besides, AE can improve patients' cardiopulmonary function and metabolic capacity to reduce obesity and metabolic diseases related to schizophrenia. However, there are still controversies about AE's effect on different cognitive areas, and whether $A E$ is having a continuous impact on cognitive function is still unclear.

INPLASY registration number: This protocol was registered with the International Platform of Registered Systematic Review and Meta-Analysis Protocols (INPLASY) on 21 October 2020 and was last updated on 21 October 2020 (registration number INPLASY2020100075).

\section{INTRODUCTION}

Review question / Objective: This metaanalysis of randomized controlled trials aim to evaluate the efficiency of adjunctive aerobic exercise for cognition in people with schizophrenia.

Condition being studied: Schizophrenia is one of the severe mental diseases. The 
prevalence rate of schizophrenia in the world is $0.5 \% \sim 1 \%$. In China, its lifetime prevalence rate is about $0.7 \%$, bringing a substantial economic burden to families and society. Cognitive dysfunction is one of the core symptoms of schizophrenia. It often appears before the psychotic symptoms such as hallucinations and delusions and runs through the disease's whole course. However, there is still a lack of effective drugs to improve cognitive function. In recent years, some studies have shown that aerobic exercise (AE) can improve the cognitive function of schizophrenic patients. After long-term aerobic exercise, the volume of the temporal lobe and hippocampus increases, and the content of brain-derived neurotrophic factor in serum increased. Besides, AE can improve patients' cardiopulmonary function and metabolic capacity to reduce obesity and metabolic diseases related to schizophrenia. However, there are still controversies about AE's effect on different cognitive areas, and whether $A E$ is having a continuous impact on cognitive function is still unclear.

\section{METHODS}

Participant or population: Adult with schizophrenia (using the diagnostic criteria of the international classification of diseases or American Diagnostic and Statistical Manual of mental disorders).

Intervention: Aerobic exercise is the leading intervention (e.g., Cycling, running).

Comparator: Schizophrenia without aerobic exercise intervention.

Study designs to be included: We will choose randomized controlled trials.

Eligibility criteria: 1.The subjects are schizophrenic patients who met the diagnostic criteria of the international classification of diseases (ICD) or American Diagnostic and Statistical Manual of mental disorders (DSM); 2.The type of trials are randomized controlled trials; 3.The intervention measures in the experimental group is aerobic exercise + essential treatment; 4.The intervention measures in the control group is essential treatment + routine exercise behavior; 5.The outcome index is the cognitive function score, The assessment tool is MATRICS Consensus Cognitive Battery (MCCB). It included seven mental sub-items: speech learning and memory, visual-spatial memory, working memory, processing speed, attention/alertness, reasoning and problem-solving ability, and social cognition. If it's not MCCB, the detection tasks used should be similar to the related sub-tasks of MCCB, or some studies show an apparent correlation.

Information sources: We will search PubMed, EMBASE, Cochrane Library, CNKI, Wanfang, VIP, CBM, and Google academic to collect randomized controlled trials related to aerobic exercise improving cognitive function in schizophrenia.

Main outcome(s): The outcome index is the cognitive function score. The assessment tool is the MATRICS Consensus Cognitive Battery (MCCB). It included seven mental sub-items: speech learning and memory, visual-spatial memory, working memory, processing speed, attention/alertness, reasoning and problem-solving ability, and social cognition. If it's not MCCB, the detection tasks used should be similar to the related sub-tasks of MCCB, or some studies show an apparent correlation.

Quality assessment / Risk of bias analysis: Two reviewers will independently assess the quality of the selected studies according to the Cochrane Collaboration's tool for randomized controlled trials. We will evaluate items in three categories: Low risk of bias, unclear bias, and high risk of bias. We will assess the following characters: random sequence generation (selection bias), allocation concealment (selection bias), blinding of participants and personnel(performance bias), incomplete outcome data(attrition bias), selective reporting(reporting bias), and so on.

Strategy of data synthesis: We will use Cochrane $Q$ and 12 statistics to assess heterogeneity. The results without 
heterogeneity $(12<50 \%, \mathrm{P}>0.1)$ were analyzed by fixed-effect model, and those with heterogeneity $(12>50 \%, \mathrm{P}<0.1)$ were analyzed by random effect model. We will use the standardized mean difference (SMD) and 95\% confidence interval (Cl) to express the difference. We will use Stata software to conduct statistical analysis.

Subgroup analysis: None.

Sensibility analysis: We will use an influence analysis to ascertain the results of the meta-analysis by excluding each of the individual studies.

Country(ies) involved: China.

Keywords: Schizophrenia; Cognition; Aerobic exercise; Meta-analysis.

Contributions of each author:

Author 1 - Du Jian.

Author 2 - Zheng Yue.

Author 3 - Liu Wei. 